\title{
Minding the Mindfulness Research in Healthcare: More Data on Diversity are Urgently Needed
}

\author{
Basant Pradhan MD \\ Cooper Medical School of Rowan University, pradhan-basant@cooperhealth.edu
}

Cooper Rowan Medical Journal: https://rdw.rowan.edu/crjcsm

Would you like to be a reviewer? Please fill in this short form to express your interest.

\section{Recommended Citation}

Pradhan, Basant MD (2020) "Minding the Mindfulness Research in Healthcare: More Data on Diversity are Urgently Needed," Cooper Rowan Medical Journal: Vol. 1 : Iss. 1 , Article 8.

DOI: 10.31986/issn.2578-3343_vol1iss1.8

Available at: https://rdw.rowan.edu/crjcsm/vol1/iss1/8

\section{(c) (7)}

This work is licensed under a Creative Commons Attribution 4.0 License.

This Editorials and Letters to the Editor is brought to you for free and open access by the Rowan University Journals at Rowan Digital Works. It has been accepted for inclusion in Cooper Rowan Medical Journal by an authorized editor of Rowan Digital Works. For more information, please contact brush@rowan.edu. 
Minding the Mindfulness Research in Healthcare: More Data on Diversity are Urgently Needed 


\title{
Minding the Mindfulness Research in Healthcare: More Data on Diversity are Urgently Needed
}

\section{In reply to article “Addressing Diversity in Mindfulness Research on Health: A Narrative Review using the ADDRESSING Framework “}

\author{
Basant Pradhan ${ }^{1,2 *}$ \\ ${ }^{1}$ Cooper Medical School of Rowan University, NJ, 401, Broadway, Camden \\ ${ }^{2}$ Department of Psychiatry, Cooper University Hospital, 1 Cooper Plaza, NJ, Camden \\ *Corresponding author: *correspondingauthor:pradhan-basant@cooperhealth.edu (Basant Pradhan)
}

\begin{abstract}
"Amongst the myriads of applications of meditative practice, mindfulness meditation can be arguably the simple yet most evidence-based type of meditative method. This simple and age-old technique emphasizes upon cultivating in the practitioner a nonjudgmental awareness on a moment-to-moment basis.

Mindfulness meditation was introduced to the American audience in the early 1990' s by Jon Kabat-Zinn, and beginning the early 2000s has seen an exponential growth trajectory that continues to this day.
\end{abstract} However, its rising popularity has given rise to considerable misinformation and misunderstanding as well."

As far as the evidence base and utility in healthcare is concerned, one sees laxity with respect to three broad and related aspects, e.g. lack of optimal standardization, scarcity of customization of the methods in the personalized medicine format and relative lack of methodologically rigorous research1. Over the last decade there has been significant progress made in the latter two areas, thanks in a large part to neuroimaging and enhanced interdisciplinary collaborative efforts. ${ }^{2}$

Although there is a great amount of diversity/variation in mindfulness with respect to the type of techniques and practices, ironically enough, the mindfulness research on diversity with respect to the psychosocial variables is grossly lacking. These are important social determinants and may be linked to the acceptability, generalizability and the clinical outcomes of the mindfulness-based interventions (MBIs) in healthcare. Thus, although substantial research supports mindfulness-related improvements in patientreported mental and physical health, ${ }^{3}$ it is clear that mindfulness research so far has over-represented the clientele from middle-to-upper class, Caucasian, and female gender. ${ }^{4}$ As mindfully described by Van Dam et al. (2018) , "During the past two decades, mindfulness meditation has gone from being a fringe topic of scientific investigation to being an occasional replacement for psychotherapy, tool of corporate wellbeing, widely implemented educational practice, and key to building more resilient soldiers." We are still in the dark about the extent to which these results may generalize to a broader, more diverse population. 
Given the replication crisis in social science and medicine, alongside numerous methodological concerns including heterogeneity, sample over-representation, data on extant mindfulness studies including trait versus state mindfulness, ${ }^{6}$ heuristically it is important to study mindfulness with respect to the important psychosocial variables including those in the underrepresented groups, which has the potential to address some of the existing issues such as generalizability. Mindfulness research has traditionally focused on traditional health outcomes, such as blood pressure or stress levels but moving forward it needs to be diversified to include non-traditional areas, such as the relationship between mindfulness, minority-status discrimination, and mental health outcomes etc. Attempts such as these will potentially be able to tap into a neglected area in terms of bridging the quality gap in mindfulness in healthcare research by investigating the link between the social determinants of the applicability of the extant mindfulness methods.

Chin, Anyanso, and Greeson offer a narrative review in this issue of the Cooper Rowan Medical Journal. ${ }^{5}$ The authors have synthesized the recent research that investigates the connection between mindfulness and health with respect to important socio-cultural parameters. Drawing from the 10-category ADDRESSING framework from the previously validated research7, they have synthesized and highlighted the mindfulness research conducted on historically underrepresented groups as both a method to summarize what has been done and to point out knowledge gaps that can be addressed in future research. Of note, the ADDRESSING framework was introduced by Hays ${ }^{7}$ as a guide to help clinicians better identify and understand the relevant cultural identities of their clients. In this editorial, my intent is to review the article and summarize what we do and do not know, while emphasizing the socio-demographic, cultural and psychosocial parameters that may influence outcomes. I will also generalize as to the various mindfulnessbased interventions (MBIs). My goals in doing so are to inform on these complex and often unstudied aspects of mindfulness so that they can be addressed in future research and hopefully pave the path for more replication and generalization of these age-old practices.

In the narrative review, using a rigorous search strategy, the authors have diligently highlighted the relationship between the impact of MBIs and many psychosocial determinants such as age, duration of practice (8-week vs 12-week practice formats), ethnicity, religion, socio-economic status, sexual orientation and developmental disability.

The authors point out the gap in knowledge as well as inconsistencies of research literature about relationship between participants' age and applicability/efficacy of the MBIs. As described by Chin and colleagues the relationship between the wellness promoting efficacy of MBIs in younger participants, particularly adolescents ( $n=132 ; x^{-}$age $=13$ years), is inconsistent with some studies finding no effects. ${ }^{1}$ Again according to Chin this reinforces the ongoing need to identify, replicate, and disseminate MBI 
formats adapted to optimally engage and benefit this population. Chin and colleagues, commenting on work by Lenze et al note that "In older-age participants experiencing high levels of anxiety-related distress and subjective cognitive dysfunction ( $\mathrm{n}=34$ ), no differences in outcomes occurred between the traditional 8week and the extended 12-week MBSR programs was noted, suggesting that more training is not necessarily better in older adults" .8 Chin et al also remind us that in another study10, older adults demonstrated significantly greater subjective positive affect and trait mindfulness than younger participants. The authors of this study referenced went on to point out that the trait mindfulness mediated the relationship between age and the positive effects. ${ }^{9}$

Based on the existing, albeit limited research, Chin et al point out that in people with developmental disabilities, such as autism spectrum disorder (ASD) and learning disorders, especially if they are high-functioning with mild to moderate impairments, MBIs may improve emotion regulation, problem behaviors, and quality of life (QoL). However, replicability and generalizability of these early findings are unclear and hence further studies are necessary to shed more light.

The authors also prudently recognize that there is a serious lack of diversity within mindfulness research with respect to religion and spirituality as the participants in most of the studies are predominantly Caucasian and Christian. Grounding mindfulness in participant' s religion or using it to enhance one' s spirituality can increase the scope and application of the mindfulness training especially with respect to spiritual development of the whole person, coping strategies, personal growth and stress reduction etc.. ${ }^{1}$ As pointed out by Chin et al there are a growing number of studies examining the effects of trait mindfulness and MBIs in people of color, which as a trend should continue to ensure that mindfulness interventions benefit the historically underserved.

One population that has significant potential to benefit from is the sexual minority group or the LGBTQIA + individuals. The few studies in this group almost exclusively focus upon white subjects, although people of color make up nearly $40 \%$ of the LGBTQIA+ population. ${ }^{10}$ Chin et al note "Future research exploring the effects of mindfulness in sexual minorities needs to account for diversity within this population, through including more non-white sexual minorities and those within the spectrum that do not identify as gay or lesbian, such as asexual or bisexual individuals." ${ }^{1}$

Training on MBIs can be costly with respect to money as well as time commitments and thus can prevent lower-income individuals from attending learning sessions. Several recent studies have explored MBIs in economically disadvantaged individuals, such as non-white, low-income, female participants. ${ }^{11}$ As noted by Chin et al "Research about MBIs in low-income individuals is promising, yet questions regarding the feasibility of standard MBIs in this population need to be explored further because effectiveness cannot be ascertained if barriers (e.g., substantial time commitment, lack of childcare, etc.) prevent many 
low-income individuals from participating in MBIs." 1

A scientific consensus is a complex process that requires a good amount of scientifically validated data amassed over a considerable amount of time and effort, with debate as well. Overall, mindfulness can reduce stress and improve mental health in diverse populations. However, fact remains that there are substantial knowledge gaps within the facets of diversity and multi-cultural samples and hence, moving forward, researchers should aim to fill the discussed gaps in research.

Modifications of existing MBIs, when guided by members of the population that the researcher is seeking to study, can enhance cultural acceptability and applicability. As noted by Chin et al, "Although mindfulness practice and research are primarily secular in the West, there is still room for research about the degree to which mindfulness is utilized as a secular versus spiritual practice and whether that results in differential health outcomes. Furthermore, web- and app-based mindfulness interventions are increasingly prevalent and utilized, though no rigorous testing of their effects has occurred." ${ }^{1}$ I agree with Chin et al that future development, testing, and implementation of these feasible, accessible, culturally-sensitive MBIs, including those adapted for portable devices, such as mobile app delivery, and those geared towards distance delivery via tele-video formats, will be the next steps to further understand the potential health benefits of mindfulness.

\section{REFERENCES}

1. Chin Gabrielle, Anyanso Vanessa;, Greeson Jeffrey. Addressing Diversity in Mindfulness Research on Health: A Narrative Review using the ADDRESSING Framework. Cooper Rowan Medical Journal. 2019;1(1):10-22.

2. Pradhan B. Yoga and Mindfulness Based Cognitive Therapy (Y-MBCT): A Clinical Guide. 2014.

3. Pradhan B K, Pinninti N R, Rathod S R, Psychotherapy . TIMBER Psychotherapy: For PTSD, Depression and Traumatic Psychosis.. Switzerland: Gesterbrasse. 2019;.

4. Vibe Michael, Bjørndal Arild, Fattah Sabina, Dyrdal Gunvor M, Halland Even, Tanner-Smith Emily E. Mindfulness-based stress reduction (MBSR) for improving health, quality of life and social functioning in adults: a systematic review and meta-analysis. Campbell Systematic Reviews. 2017;13(1):1-264.

5. Dam N T, Van, Vugt M K, Van, Vago D R. Mind The Hype: A Critical Evaluation and Prescriptive Agenda for Research on Mindfulness and Meditation. Perspect Psychol Sci. 2018;13(1):36-61. 
6. Waldron Elizabeth M., Hong Sunghyun, Moskowitz Judith T., Burnett-Zeigler Inger. A Systematic Review of the Demographic Characteristics of Participants in US-Based Randomized Controlled Trials of Mindfulness-Based Interventions. Mindfulness. 2018;9(6):1671-1692.

7. Hays P.Addressing Cultural Complexities in Practice: A FrameworkforClinicians and Counselors. Washington, DC, US: American Psychological Association; 2001.

8. Johnson Catherine, Burke Christine, Brinkman Sally, Wade Tracey. Effectiveness of a school-based mindfulness program for transdiagnostic prevention in young adolescents. Behaviour Research and Therapy. 2016;81:1-11.

9. Lenze Eric J., Hickman Steven, Hershey Tamara, et al. Mindfulness-based stress reduction for older adults with worry symptoms and co-occurring cognitive dysfunction. International Journal of Geriatric Psychiatry. 2014;29(10):991-1000.

10. Shook Natalie J., Ford Cameron, Strough JoNell, Delaney Rebecca, Barker David. In the moment and feeling good: Age differences in mindfulness and positive affect.. Translational Issues in Psychological Science. 2017;3(4):338-347.

11. The Williams Institute. 2016. 